University of Nebraska - Lincoln

DigitalCommons@University of Nebraska - Lincoln

Faculty Publications: Materials Research

Science and Engineering Center

Materials Research Science and Engineering

Center

February 2005

\title{
High-Anisotropy Nanocluster Films for High-Density Perpendicular Recording
}

\author{
David J. Sellmyer \\ University of Nebraska-Lincoln, dsellmyer@unl.edu \\ Minglang Yan \\ University of Nebraska - Lincoln, myan@unlserve.unl.edu \\ Yinfan Xu \\ University of Nebraska - Lincoln, yxu2@unl.edu \\ Ralph Skomski \\ University of Nebraska-Lincoln, rskomski2@unl.edu
}

Follow this and additional works at: https://digitalcommons.unl.edu/mrsecfacpubs

Part of the Materials Science and Engineering Commons

Sellmyer, David J.; Yan, Minglang; Xu, Yinfan; and Skomski, Ralph, "High-Anisotropy Nanocluster Films for High-Density Perpendicular Recording" (2005). Faculty Publications: Materials Research Science and Engineering Center. 17.

https://digitalcommons.unl.edu/mrsecfacpubs/17

This Article is brought to you for free and open access by the Materials Research Science and Engineering Center at DigitalCommons@University of Nebraska - Lincoln. It has been accepted for inclusion in Faculty Publications: Materials Research Science and Engineering Center by an authorized administrator of DigitalCommons@University of Nebraska - Lincoln. 


\title{
High-Anisotropy Nanocluster Films for High-Density Perpendicular Recording
}

\author{
David J. Sellmyer, Minglang Yan, Yingfan Xu, and Ralph Skomski
}

\begin{abstract}
This paper reports results on the synthesis and magnetic properties of $L 1_{0}: X$ nanocomposite films, where $\mathrm{L1}_{0}=\mathrm{FePt}$, CoPt, and $\mathrm{X}=\mathrm{C}$, Ag, etc. Two fabrication methods are discussed: nonepitaxial growth of oriented perpendicular media, and monodispersed nanoparticle-assembled films grown with a gas-aggregation source. The magnetic properties are controllable through variations in the nanocluster properties and nanostructure. The films show promise for development as recording media at extremely high areal densities.
\end{abstract}

Index Terms-High-anisotropy, $\mathrm{L1}_{0}$ phase, nanoclusters, perpendicular recording.

\section{INTRODUCTION}

$\mathbf{R}$ ECENTLY, there has been great interest in $\mathrm{L}_{0}$-structure nanocluster films including $\mathrm{FePt}$ and $\mathrm{CoPt}$ for extremely high-density recording (EHDR) media. The requirement of noise reduction for EHDR at areal densities approaching $1 \mathrm{~Tb} / \mathrm{in}^{2}$ imposes the need for grain sizes in the range $4-5 \mathrm{~nm}$ with a narrow size distribution. Present media, of the CoCrPtX type, suffer because the thermal stability factor, $\xi \equiv K_{u} V^{*} / k_{B} T$, where $K_{u}$ is the anisotropy constant and $V^{*}$ an activation volume, becomes less than about 50 which is required for 10-year storage. FePt and CoPt have large values of $K_{u}\left(\sim 5-7 \times 10^{7} \mathrm{erg} / \mathrm{cm}^{3}\right)$, and thus have the potential to serve as building blocks for thermally stable $\mathrm{Tb} / \mathrm{in}^{2}$ media.

Recent modeling results [1] for future $1 \mathrm{~Tb} / \mathrm{in}^{2}$ perpendicular media have suggested approximate media parameter values of $d \cong 4 \mathrm{~nm}, H_{k} \cong 25 \mathrm{kOe}, M_{s} \cong 750 \mathrm{emu} / \mathrm{cm}^{3}, \sigma / d \cong 0.07$, and $\delta \cong 20 \mathrm{~nm}$, where the parameters represent average grain size, anisotropy field, saturation magnetization, normalized rms deviation of grain size, and film thickness, respectively. In designing potential $\mathrm{L} 1_{0}$ nanocluster films with appropriate properties, it is useful to consider the relevant properties of bulk $\mathrm{L} 1_{0}$ phases, as in Table I.

It is clear that the $M_{s}$ and $H_{k}$ values of these $\mathrm{L} 1_{0}$ compounds, or modifications thereof, are in the correct range for consideration as EHDR media.

A huge literature is developing on the growth and magnetic properties of $\mathrm{L} 1_{0}$ films and their potential for EHDR. We cannot review this work in the present paper, but refer the reader to several earlier reviews and conference sessions or symposia on high-anisotropy films [2]-[8]. Along with the experimental

Manuscript received July 8, 2004. This work was supported by INSIC, NSFMRSEC, W. M. Keck Foundation, ARO, and CMRA.

The authors are with the Center for Materials Research and Analysis and Department of Physics and Astronomy, University of Nebraska, Lincoln, NE 68588-0113 USA (e-mail: dsellmyer@unl.edu; myan@unlserve.unl.edu; yxu2@unl.edu; rskomski@unlserve.unl.edu).

Digital Object Identifier 10.1109/TMAG.2004.838072
TABLE I

Magnetic Properties of Selected L $1_{0}$ Compounds

\begin{tabular}{cccc}
\hline Compound & $\mathrm{M}_{\mathrm{s}}(\mathrm{emu} / \mathrm{cc})$ & $\mathrm{K}_{\mathrm{u}}\left(10^{7} \mathrm{erg} / \mathrm{cc}\right)$ & $\mathrm{H}_{\mathrm{k}}(\mathrm{kOe})$ \\
\hline $\mathrm{FePt}$ & 1140 & 6.6 & 116 \\
$\mathrm{CoPt}$ & 800 & 4.9 & 123 \\
$\mathrm{FePd}$ & 1100 & 1.8 & 33 \\
\hline
\end{tabular}

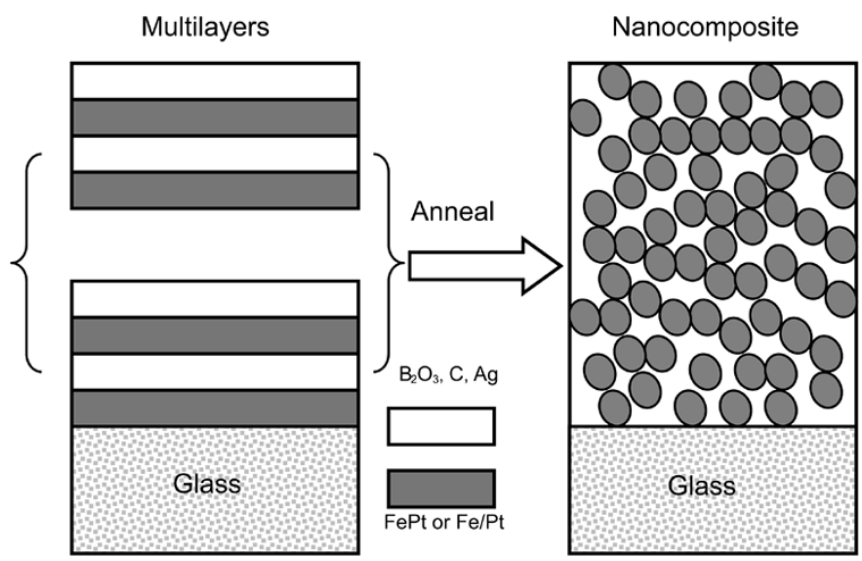

Fig. 1. Preparation method of nonepitaxial growth $\mathrm{L}_{0}$-phase FePt:X nanocomposite films.

work, a number of recent simulations have appeared and these are important for designing and optimizing perpendicular media for densities approaching $1 \mathrm{~Tb} / \mathrm{in}^{2}$ [9]-[12].

In this paper, we report on recent efforts to fabricate $\mathrm{L} 1_{0}: \mathrm{X}$ and related nanocomposite cluster films, where $\mathrm{L}_{0}=\mathrm{FePt}$, $\mathrm{CoPt}$ and $\mathrm{X}=\mathrm{C}, \mathrm{Ag}, \mathrm{B}_{2} \mathrm{O}_{3}$, etc., and on their suitability for EHDR. We have developed two novel synthetic methods: nonepitaxial growth of oriented perpendicular media, and nanoparticle-assembled films grown with a gas-aggregation source. The methods, the resulting magnetic properties, and their advantages and limitations are described below.

\section{Synthetic MethodS}

\section{A. Nonepitaxial Growth of Perpendicular Media}

The nonepitaxially grown oriented $\mathrm{L} 1_{0}$ nanocomposite films were prepared by multilayer deposition plus annealing. Fig. 1 shows the initial and final structures schematically for preparation of the $\mathrm{FePt}: \mathrm{X}\left(=\mathrm{Ag}, \mathrm{C}, \mathrm{B}_{2} \mathrm{O}_{3}\right.$, etc. $)$ oriented $\mathrm{L} 1_{0}$ films. $\mathrm{FePt} / \mathrm{X}$ or $\mathrm{Fe} / \mathrm{Pt} / \mathrm{X}$ layers were deposited individually 


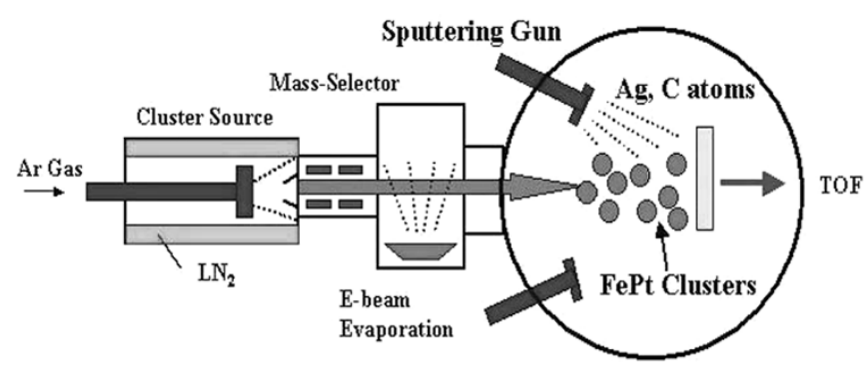

Cluster Size d $\sim 3-12 \mathrm{~nm}$

Fig. 2. Schematic drawing of a cluster deposition system.

on $\mathrm{Si}$ wafer or glass substrates by magnetron sputtering at room temperature and then annealed in vacuum for 5 to $30 \mathrm{~min}$ at various temperatures. The as-deposited multilayers were broken up and nanocomposite cluster films were formed with $\mathrm{L} 1_{0}$-phase FePt grains embedded in the nonmagnetic matrix. The orientation of the $c$ axis of the fct $\mathrm{L}_{0}$-phase nanoparticles is strongly dependent on initial multilayering of $\mathrm{Fe} / \mathrm{Pt}$ as well as dimensions of the $\mathrm{Fe}, \mathrm{Pt}$, and $\mathrm{X}$ individual layers. Examples of this will be shown in Section III.

\section{B. Monodispersed Nanoparticle-Assembled Films}

Formation of metal clusters by gas aggregation, in which metal atoms are evaporated or sputtered into a cooled inert gas flow at relatively high pressure, has been established in several laboratories in the last decade. By repeated collisions with the carrier gas, the supersaturated metal vapor nucleates and forms clusters. The mechanism of cluster formation can be explained with homogeneous and heterogeneous nucleation theories. The gas aggregation methods have been applied extensively to produce small clusters of metals such as zinc, copper, silver, etc. In some cases, this method was used in combination with a mass filter such as a quadruple or a time-of-flight spectrometer. The metal vapor for cluster source can be produced by either thermal evaporation or sputter discharge [13].

The advantages of the sputtering-based cluster source are: 1) it can produce a very large range of mean cluster sizes from 200 to 15000 atoms per cluster; 2) it has a high degree of ionization from $20 \%$ to $50 \%$, depending on the target materials; and 3 ) a wide variety of elements and alloys can be used as source materials. Usually the deposition rate is about $3 \AA / \mathrm{s}$ for the total flux of the cluster beam.

Fig. 2 shows a schematic drawing of our cluster-deposition system. It consists of a gas-aggregation source with a liquid $\mathrm{N}_{2}$-cooled sheath and sputtering gun, a quadrupole mass selector to control the dispersion of particle diameters, a chamber including e-beam evaporation for particle coating, and a deposition chamber containing the substrate and one or more sputtering guns to deposit the matrix $(\mathrm{C}, \mathrm{Ag}$, etc.).

\section{RESULTS AND DISCUSSION}

\section{A. Nonepitaxially Grown (001) Textured FePt: X Films}

Under normal growth conditions, $\mathrm{L} 1_{0}$-phase $\mathrm{FePt}$ and $\mathrm{FePt}$ based films often possess (111) preferred or random orientations. In order to control crystal orientation, the epitaxial

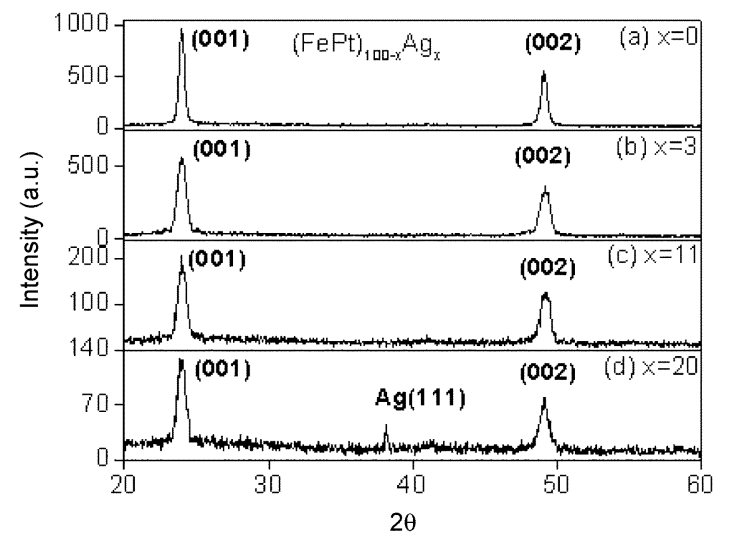

Fig. 3. XRD patterns of FePt: Ag films with different Ag content. Films were deposited directly on Si wafer and annealed at $600{ }^{\circ} \mathrm{C}$ for $10 \mathrm{~min}$. Film thickness is $10 \mathrm{~nm}$.

growth of FePt film is needed to obtain oriented films. Common methods to obtain the $c$ axis normal to the film plane [ $(001)$ texture] are to use seed or buffer layers between a single-crystal or textured substrate, such as $\mathrm{MgO}$ or $(001) \mathrm{Cr}$, and the $\mathrm{FePt}$ or FePt-based layer. Recently, highly (001) textured $\mathrm{L}_{0} \mathrm{FePt}$ [14], [15] and FePt-based films [16] have been obtained by a nonepitaxial growth method. Yan and co-workers obtained (001) textured FePt and FePt-based films by using multilayer deposition plus annealing. As explained in [14], [17], and [18], it is found that orientations of FePt grains are affected by many preparation parameters, such as initial as-deposited film structure, composition, annealing time, and total film thickness. Although multilayering of $\mathrm{Fe}$ and $\mathrm{Pt}$ in the initial structure qualitatively can be seen to promote growth of (001)-textured $\mathrm{L} 1_{0} \mathrm{FePt}$ grains, the complexity of the data on film growth have precluded any simple explanations.

The effect of matrix concentration on orientation, intergranular interactions, and magnetic properties has been investigated for $\mathrm{L}_{0} \mathrm{FePt}$ : Ag nanocomposite films [17]. The Ag contents varied from 0 to 20 vol\%. As shown in Fig. 3, all films clearly show the (001) superlattice peaks indicating that the Ag content (matrix) has a relatively small effect on orientation.

A key aspect of the magnetism of nanocomposite films is the relation between intergranular interactions, the hysteresisloop shape, and spatial correlations. Fig. 4 shows the hysteresis loop slope, correlation length, and coercivity of the FePt: Ag nanocomposite films with various $\mathrm{FePt}$ contents. Average interaction-domain sizes (correlation lengths) were deduced from MFM images. The coercivities and the hysteresis-loop slope parameter $\alpha=4 \pi d M / d H\left(H_{c}\right)$ were determined from the hysteresis loops. With increasing FePt content the hysteresis-loop slope and the correlation length increase, whereas the coercivity decreases. The Ag increases the separation between the particles, thereby effectively exchange-decoupling the grains.

Hysteresis loops for an $\mathrm{L}_{0}$ nanocomposite $\mathrm{FePt}$ : $\mathrm{C}$ film are shown in Fig. 5. Inset are the XRD patterns of the same FePt:C film, on which (001), (002) peaks appear indicating that FePt grains are (001) oriented. The full width at half maximum (FWHM), obtained from the rocking curve of (001) peak, is $1.68^{\circ}$ confirming a high degree of (001) texturing. The loop shows perpendicular anisotropy with square shape 


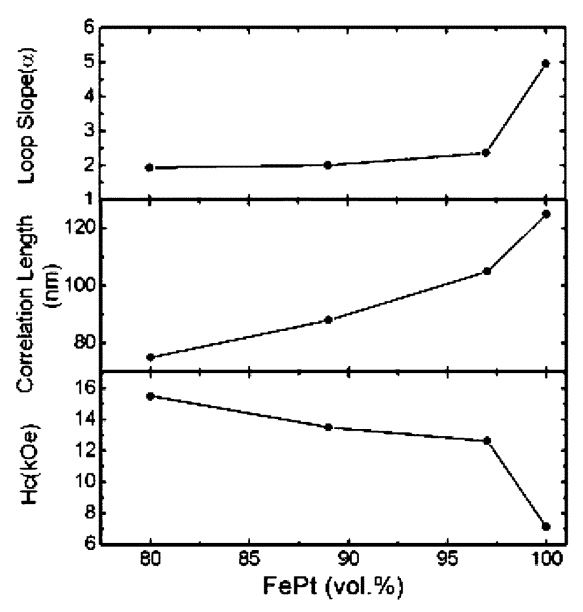

Fig. 4. Hysteresis loop slope, correlation length and coercivity for various $\mathrm{FePt}$ contents (vol.\%) in 10-nm-thick FePt: Ag films.

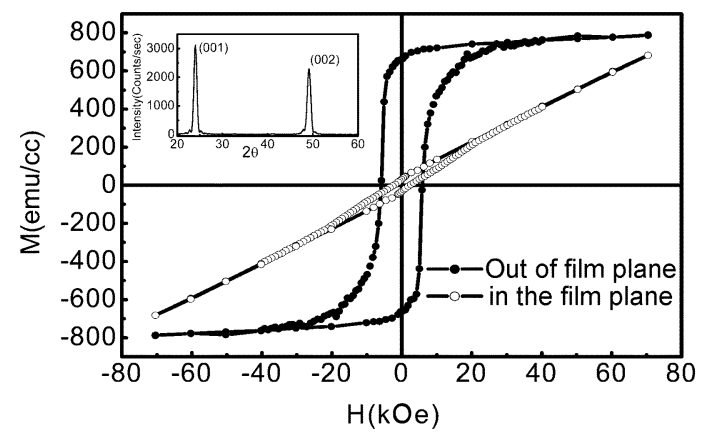

Fig. 5. Hysteresis loop of FePt:C film ( $\sim 55$ vol.\% FePt). Film deposited directly on Si wafer and annealed at $550{ }^{\circ} \mathrm{C}$ for $5 \mathrm{~min}$. Film thickness is $16 \mathrm{~nm}$. Inset is the XRD pattern of this sample.

in perpendicular direction due to the enhanced preferential FePt $\mathrm{L1}_{0}$ (001) texture [18]. The perpendicular loop shows large coercivity $\left(H_{c}=6.2 \mathrm{kOe}\right)$ and high remanence ratio $(S=0.9)$.

The nanostructure of nonepitaxially grown $\mathrm{L} 1_{0} \mathrm{FePt}: \mathrm{C}$ thin film was characterized by transmission electron microscopy (TEM). The results show FePt grains with uniform size less than $5 \mathrm{~nm}$ are embedded in the carbon matrix and appear to be well isolated.

\section{B. Double-Layered FePt: C/FeCoNi Perpendicular Recording Media}

For recording studies, a nonepitaxially grown, double-layered thin-film medium of nanocomposite $\mathrm{FePt}$ : $\mathrm{C}$ with a FeCoNi soft underlayer (SUL) was fabricated and investigated [18]. The 150-nm FeCoNi SUL was first deposited on thermally oxidized $\mathrm{Si}$ substrates. The saturation magnetization flux density $\left(B_{s}\right)$ for this layer is approximately $20 \mathrm{kG}$. The $16-\mathrm{nm}$ FePt : C layer was then deposited on the SUL at room temperature by a multilayer deposition technique and subsequent annealing. A 5-nm Ta barrier layer was introduced between SUL and $\mathrm{FePt}$ : $\mathrm{C}$ layer to reduce the diffusion. A cross sectional TEM image verifies that this medium consists of nanocomposite FePt: C layer with 5-nm FePt nanoparticles embedded in a C matrix [18].
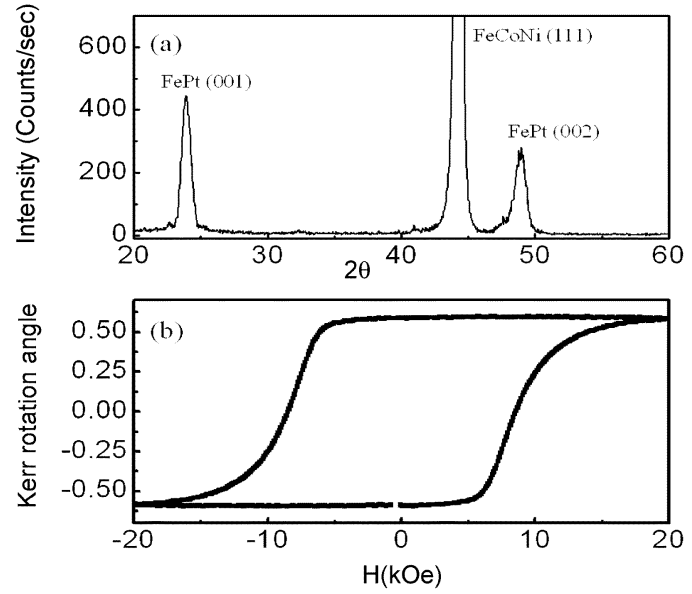

Fig. 6. (a) XRD pattern and (b) hysteresis loop of FePt: $\mathrm{C}$ double-layered media. Loop measured by polar-Kerr effect at room temperature.

Fig. 6 shows the (a) XRD pattern and (b) hysteresis loop of FePt : C double-layered nanocomposite thin-film medium. Only the soft underlayer FeCoNi (111) peak and the $\mathrm{L}_{0} \mathrm{FePt}(001)$ and (002) peaks appear in the XRD pattern. This means that the preferred crystal orientation of $\mathrm{L} 1_{0} \mathrm{FePt}: \mathrm{C}$ nanocomposite film is successfully obtained on this SUL by nonepitaxial growth. The polar-Kerr measurement shows a square loop that is only sensitive to the top layer; the Kerr effect data shown in this loop give the coercivity $H_{c}=8.5 \mathrm{kOe}$, nucleation field $H_{n}=$ $5.65 \mathrm{kOe}$, remanence ratio $S=1$, and loop slope (at $H_{c}$ ) $\alpha=3.3$, respectively. Preliminary static read/write tests have been performed on these films with the result that bits could be written but only with abnormally large write currents. This likely is the result of rather large saturation fields as seen in Fig. 6.

\section{Nanocluster-Assembled L1 $1_{0}$ Films}

1) Size Distribution and Nanostructure of Clusters: In this section, we focus on $\mathrm{FePt}(\mathrm{CoPt})$ clusters which have the highanisotropy $\mathrm{L} 1_{0}$ phase after annealing [19]-[21]. For the TEM observations, FePt, CoPt nanoclusters were directly deposited onto carbon-coated films supported by $\mathrm{Cu}$ grids.

The cluster size was controlled in a range from 3 to $6 \mathrm{~nm}$ by adjusting the preparation parameters such as sputtering power, $\mathrm{Ar}-\mathrm{He}$ gases flow, and gas pressure, etc. Uniform cluster size distribution with standard deviation $\sigma / d<0.1$ is obtained. The size distribution can be further improved if desired by applying a mass selector. Fig. 7 shows the TEM image of as-deposited FePt nanoclusters. The corresponding cluster size distribution counted by more than 200 clusters from lower magnification TEM pictures is also included. Spherical clusters with an average size of $5.3 \mathrm{~nm}$ fitted with Gaussian distribution (with standard deviation $\sigma=0.46 \mathrm{~nm}, \sigma / d=0.09$ ) were obtained with sputtering power of $200 \mathrm{~W}$ and $\mathrm{Ar} / \mathrm{He}$ gas flow of $400 / 200 \mathrm{sccm}$. High-resolution TEM image reveals some facet feature of the FePt clusters as shown in Fig. 8. An enlarged FePt cluster image shows the lattice fringes, indicating the single crystal structure. Similar results were also obtained for CoPt nanoclusters.

Fig. 9 gives two examples of cluster film structure: clusters are embedded in a matrix by co-deposition (a) or isolated by 

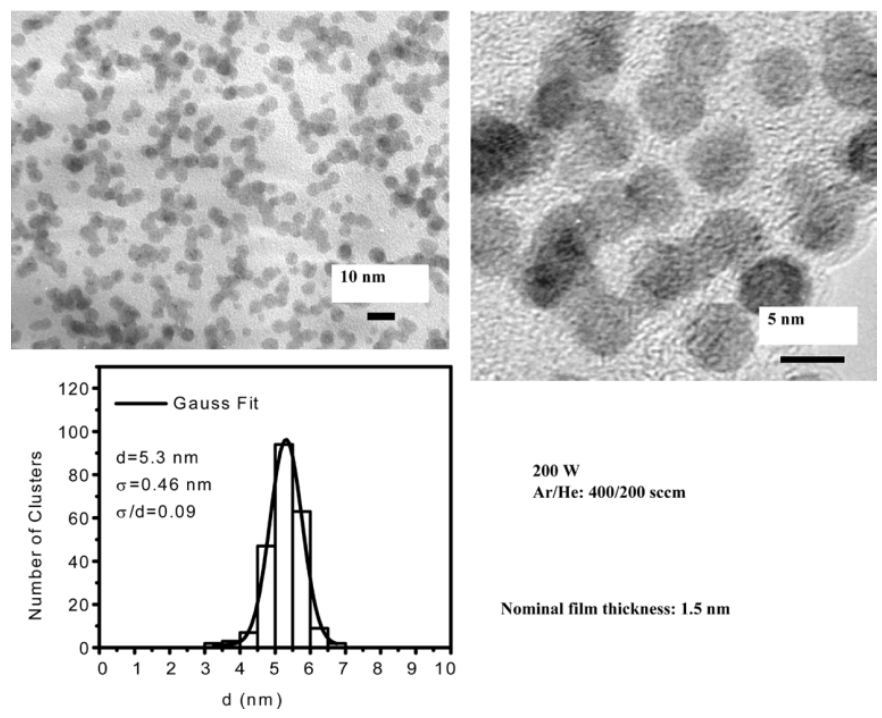

$200 \mathrm{~W}$

Ar/He: 400/200 scem

Nominal film thickness: $1.5 \mathrm{~nm}$

Fig. 7. TEM images of FePt clusters with cluster size distribution. Right: larger magnification of the clusters.

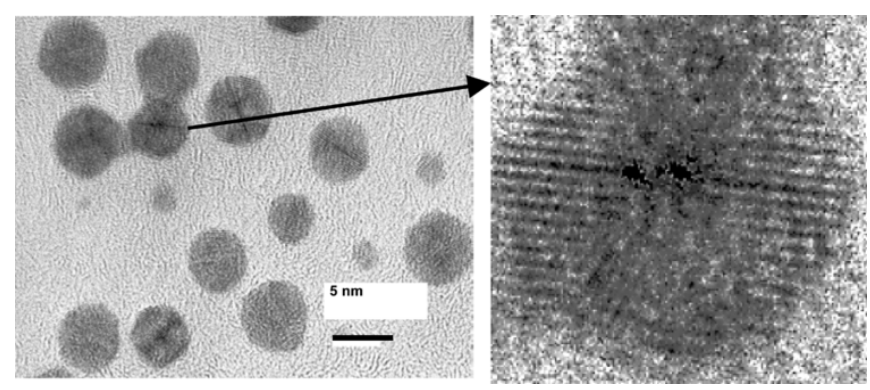

Fig. 8. HRTEM images of FePt clusters with facet feature. Right: an enlarged cluster showing lattice fringes.

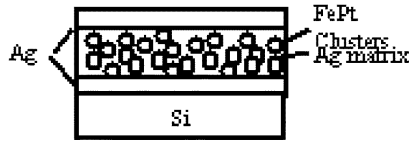

(a)

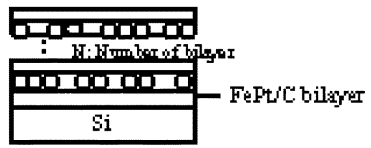

(b)
Fig. 9. Cluster film structures. (a) Co-deposition. (b) Multilayers.

the matrix via multilayers (b). The ability of the deposition technique to independently control these parameters makes it ideal for systematic studies of magnetic clusters. In this section, some recent studies on FePt: $\mathrm{Ag}$ and $\mathrm{FePt}: \mathrm{C}$ cluster films are presented.

2) FePt:Ag Cluster Films: FePt:Ag cluster films are deposited with three-layer structure $[\mathrm{Ag}(2 \mathrm{~nm}) / \mathrm{FePt}(12 \mathrm{~nm}) / \mathrm{Ag}(2$ $\mathrm{nm})$ ] or co-sputtering FePt clusters and Ag atoms on Si substrate. The mean diameter of the clusters is about $4.4 \mathrm{~nm}$. For the three-layer films, the magnetic layer thickness is about $12 \mathrm{~nm}$, which is nearly three layers of FePt clusters [19]. X-ray diffraction (XRD) measurement confirmed the ordering of $\mathrm{L}_{0}$ phase by post-deposition annealing. The as-deposited film showed a broad FePt fcc (111) peak and Ag fcc (111) and (200) peaks. After annealing at higher temperature, the fct-(200) and (002) peaks are well-separated, and the intensity of fct-(001) peak increased with the increase of annealing temperature, indicating that the degree of $\mathrm{L} 1_{0}$ ordering increased with the annealing

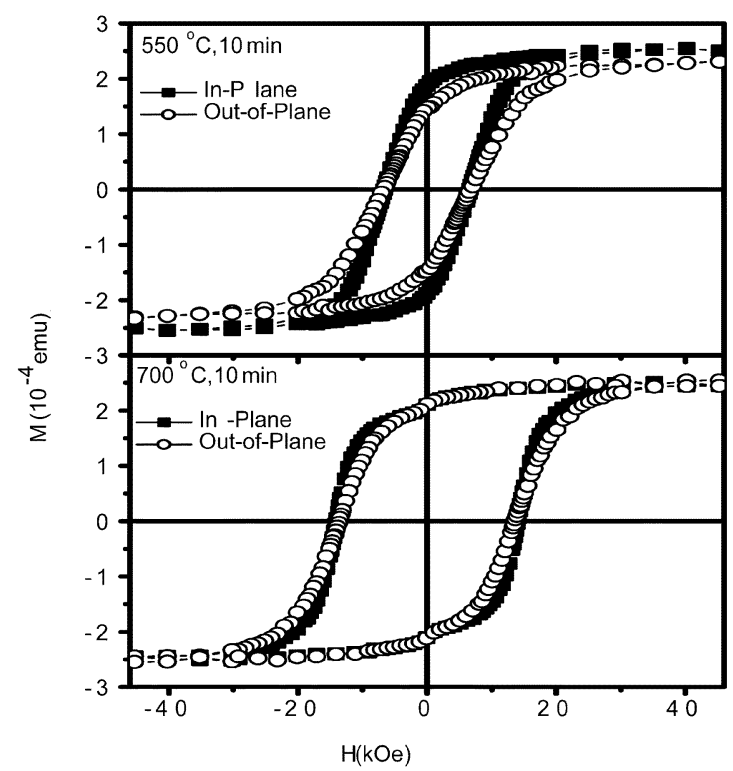

Fig. 10. Hysteresis loops of FePt: Ag cluster films annealed at $550{ }^{\circ} \mathrm{C}$ and $700{ }^{\circ} \mathrm{C}$ for $10 \mathrm{~min}$.

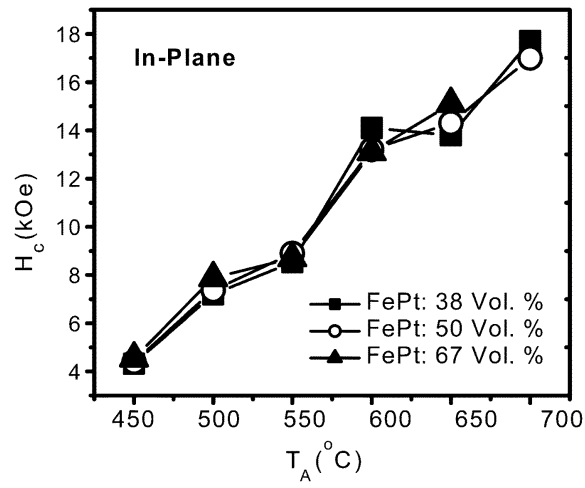

Fig. 11. Effect of annealing temperature on coercivities for FePt: Ag cluster films.

temperature. In all above films, the orientation of the fct FePt clusters is random.

Magnetic properties of the FePt:Ag cluster films were measured by superconducting quantum interference device (SQUID). Fig. 10 shows the room temperature hysteresis loops of the FePt: Ag films annealed at $550{ }^{\circ} \mathrm{C}$ and $700{ }^{\circ} \mathrm{C}$ for 10 min. Both in-plane ( $\mathrm{H} / /$ film plane) and perpendicular $(\mathrm{H} \perp$ film plane) loops are presented. The perpendicular coercivity is almost the same as the in-plane coercivity, indicating that the easy axis of clusters is distributed randomly. This result is consistent with that obtained by XRD measurement. Fig. 11 shows the dependence of the coercivity with annealing temperature for co-sputtered FePt: Ag films. The coercivity increases linearly with the increase of annealing temperature. This can be understood on the basis of the increase of the degree of $\mathrm{L}_{0}$ ordering which leads to the large $K_{u}$ of the fct phase. Note that the coercivity more than $4 \mathrm{k}$ Oe can be achieved just by annealing the film at $450^{\circ} \mathrm{C}$ for $10 \mathrm{~min}$, which otherwise could be obtained by annealing samples at $550{ }^{\circ} \mathrm{C}$ or higher for $\mathrm{FePt}$ cluster films without $\mathrm{Ag}$ addition. This result suggests that addition of $\mathrm{Ag}$ in $\mathrm{FePt}$ cluster films can lower the $\mathrm{L} 1_{0}$ ordering 

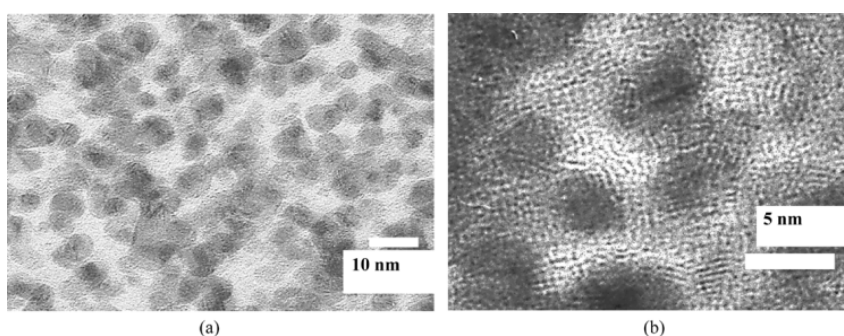

Fig. 12. TEM images of FePt : C cluster film with 45 vol. $\% \mathrm{C}$, annealed at $650{ }^{\circ} \mathrm{C}$ for $10 \mathrm{~min}$.

temperature and increase the coercivity of the cluster films significantly.

3) FePt: C Cluster Films: FePt: C nanocluster films were prepared with a multilayer method in which FePt cluster layers and $\mathrm{C}$ layers were alternately deposited onto a Si substrate. C serves to isolate the clusters and to avoid cluster-aggregation during thermal annealing. The average FePt cluster size is about $4.5 \mathrm{~nm}$. The nominal thickness of each cluster layer is about $1 \mathrm{~nm}$, so that the FePt clusters can be well separated with $\mathrm{C}$ layer. The thickness of $\mathrm{C}$ layer was adjusted with $\mathrm{C}$ volume fraction changing from $7 \%$ to $45 \%$ [22].

Fig. 12 shows the TEM plan-view images for the FePt:C film with 45 vol. $\% \mathrm{C}$ annealed at $650{ }^{\circ} \mathrm{C}$ for $10 \mathrm{~min}$. It can be seen that FePt clusters are embedded in $\mathrm{C}$ matrix. Shown in Fig. 12(b) is the high-resolution TEM image. It shows that single FePt clusters with diameter about $4.5 \mathrm{~nm}$ were well separated by amorphous $\mathrm{C}$ matrix; the cluster growth during high temperature annealing has been remarkably reduced with the isolation of higher volume fraction of $\mathrm{C}$.

XRD measurements confirmed the ordering of the $\mathrm{L} 1_{0}$ structure [22]. The hysteresis loops of FePt: C cluster films were measured at low temperature down to $10 \mathrm{~K}$. The coercivity dependence on temperature for FePt : C cluster films with 45 vol.\% $\mathrm{C}$ was measured from 10 to $300 \mathrm{~K}$. The coercivity decreases with increase of measuring temperature, e.g., dropping from $19 \mathrm{kOe}$ at $10 \mathrm{~K}$ to about $13 \mathrm{kOe}$ at $300 \mathrm{~K}$ for a film annealed at $750{ }^{\circ} \mathrm{C}$ for $10 \mathrm{~min}$. This may be caused by a contribution of intrinsic temperature dependence of the anisotropy and magnetization, and thermal activation effects [5]. The $\Delta m$ curve measured for the FePt : C film with 45 vol. $\% \mathrm{C}$ annealed at $625^{\circ} \mathrm{C}$ for $10 \mathrm{~min}$ shows a small negative value of $\Delta m$ peak $(-0.17)$; this suggests only relatively weak dipolar interactions.

4) Dynamics of Magnetization Reversal in FePt: C Cluster Films: Understanding the magnetization reversal behavior of media is of essential importance to control and design new media with desired properties and good thermal stability. We carried out a set of moment decay measurements for FePt : C films with 45 vol.\% C. The data were fitted with the Sharrock formula [23], and the thermal stability factor and anisotropy constant were extracted from the fitting parameters. The detailed experiment and fitting procedures can be found in [22]-[24].

Fig. 13 shows the annealing temperature effect on the thermal stability factor $K_{u} V^{*} / k_{B} T$ and coercivity prefactor $H_{0}$ for the FePt:C films with 45 vol.\% C. $K_{u} V^{*} / k_{B} T$ increases linearly with $T_{A}$ except for the point at $675{ }^{\circ} \mathrm{C}$ that might be

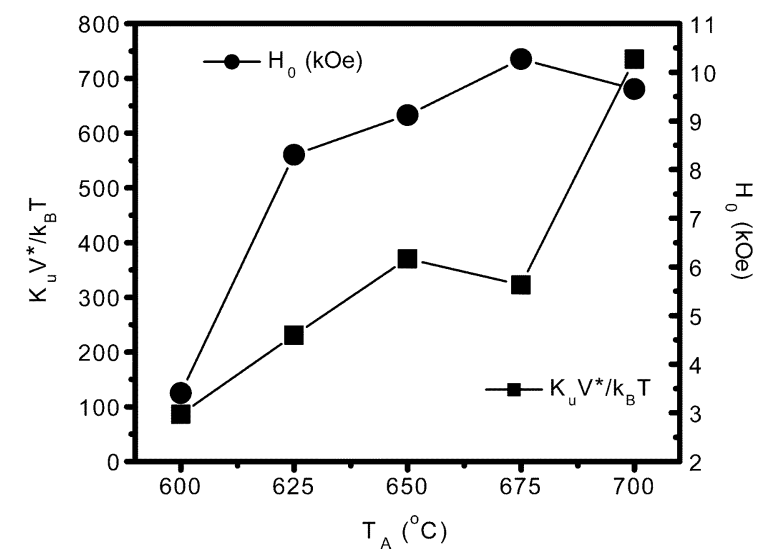

Fig. 13. Effect of annealing temperature on thermal stability and $H_{0}$ of FePt : C cluster films with 45 vol. $\% \mathrm{C}$.

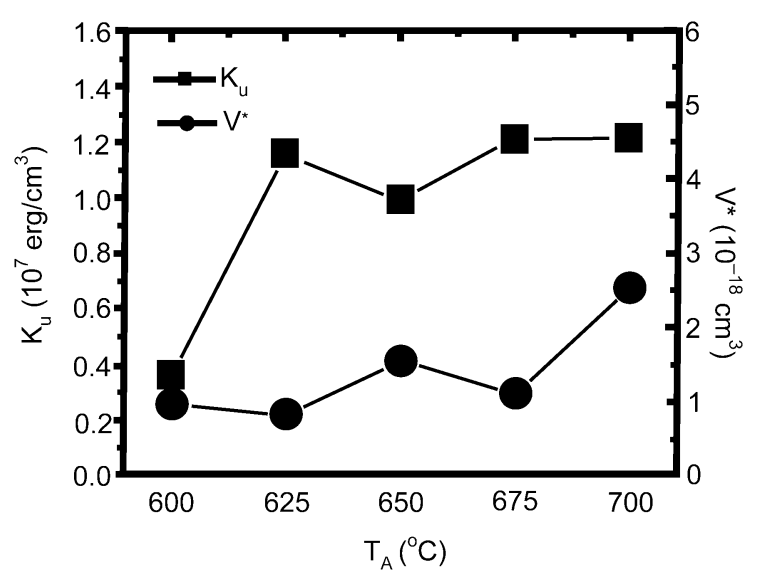

Fig. 14. Effect of annealing temperature on magnetic anisotropy constant and activation volume of FePt : C cluster films with 45 vol.\% C.

caused by either experimental error or $V^{*}$ being unusually small. Since $K_{u}$ would be constant after the completion of $\mathrm{L} 1_{0}$ ordering, the further increase of $K_{u} V^{*} / k_{B} T$ with $T_{A}$ is mainly due to the increase of $V^{*}$. As shown in Fig. 14, $K_{u}$ is about $1.2 \times 10^{7} \mathrm{erg} / \mathrm{cm}^{3}$ for $T_{A}>625^{\circ} \mathrm{C}$. It is noted that $V^{*}$ is larger than the grain volume $V$, which suggests exchange interactions between clusters and/or incoherent magnetization reversal.

5) Dilute FePt:C Cluster Film: Stoner-Wohlfarth-Like Behavior: Understanding the magnetic properties of individual clusters is of great interest for exploring FePt clusters as a media for EHDR. Dilute FePt : C cluster films with 5 vol.\% FePt were prepared by the multilayer method as described earlier. TEM images of this film showed well isolated clusters with single crystal $\mathrm{L}_{0}$ structure. Shown in Fig. 15 is the temperature dependence of coercivity for the $\mathrm{FePt}$ : C films annealed at $700{ }^{\circ} \mathrm{C}$ for 10,30 , and $60 \mathrm{~min}$, respectively. High coercivities $(>21 \mathrm{kOe})$ at room temperature were observed and they increased to about $40 \mathrm{kOe}$ at low temperature. This result indicates the high degree of $\mathrm{L} 1_{0}$ ordering after annealing for a longer time ( $>10 \mathrm{~min}$ ). Assuming these isolated clusters are noninteracting and the formula $H_{c}=0.48 H_{k}$ can be applied, an anisotropy field of $83 \mathrm{kOe}$ is estimated. 


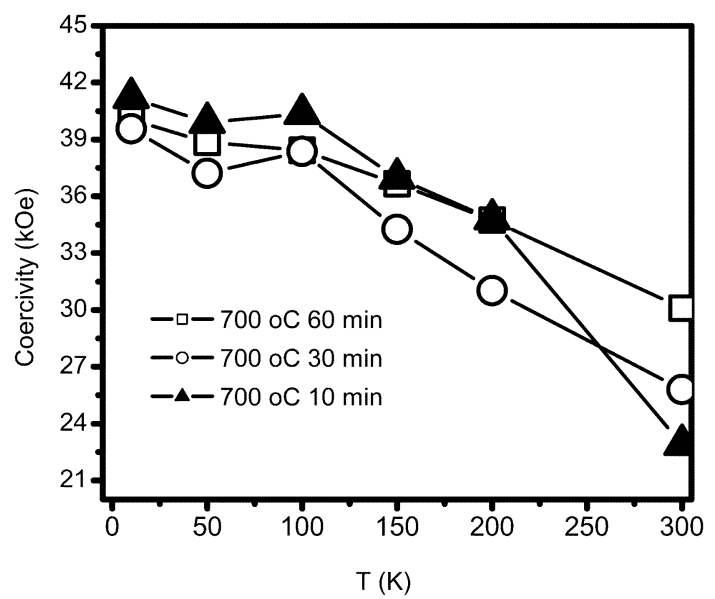

Fig. 15. Temperature dependence of coercivity of dilute FePt : C cluster films with 5 vol. $\% \mathrm{FePt}$ annealed at $700{ }^{\circ} \mathrm{C}$ for 10,30 , and $60 \mathrm{~min}$.

\section{CONCLUSION}

In the nonepitaxially grown films, the development of excellent (001) texture of the clusters is quite interesting. The multilayering of the $\mathrm{Fe} / \mathrm{Pt}$ and individual layer thicknesses, annealing temperature and time, total film thickness, and alloy composition, etc., appear to play important roles [14], [16]. A detailed explanation of the origins of the $c$-axis orientation, the grain-size distribution, cluster structure with a possible shell with different properties, degree of $\mathrm{L} 1_{0}$ ordering, intercluster exchange interactions, maximum packing fraction, control of $H_{\text {sat }}$, and magnetization reversal mechanism all are challenges requiring further research.

In the cluster-assembled films, estimates of the anisotropy $K_{u}=H_{k} M_{s} / 2$ range from about $1 \times 10^{7} \mathrm{erg} / \mathrm{cm}^{3}$ to $4.7 \times 10^{7} \mathrm{erg} / \mathrm{cm}^{3}$, depending on the system, packing fraction, annealing temperature, etc. The larger value gives an indication of the high degree of perfection and $\mathrm{L}_{0}$ ordering in the clusters, while the smaller value suggests that control of the anisotropy and coercivity to appropriate values for writing is possible.

Simulation studies have shown that both intercluster exchange interactions and incoherent reversal (due to a core/shell structure) can lead to $H_{c}$ values decreased from ideal Stoner-Wohlfarth values.

Studies are underway on coating the FePt particles with thin molecular layers to impede exchange interactions at high packing fractions. In addition, work is being undertaken to control the orientation of the clusters after forming the $\mathrm{L} 1_{0}$ phase. Pseudo-binary alloys of the form $\mathrm{Fe}_{1-x} \mathrm{Ni}_{x} \mathrm{Pt}$ are being studied to control the $K_{u}$ and $H_{c}$ values.

Clearly the FePt (and $\mathrm{CoPt}$ ) nanoclusters studied are thermally stable, and have a narrow size distribution and controllable coercivities. Their properties are similar to those required for magnetic recording at extremely high densities. Further re- search will determine whether they can be used practically for standard or heat-assisted perpendicular recording.

\section{REFERENCES}

[1] R. Gustafson, private communication, 2004

[2] D. J. Sellmyer, M. Yu, R. A. Thomas, Y. Liu, and R. D. Kirby, "Nanoscale design of films for extremely high density magnetic recording," Phys. Low-Dimensional Structures, vol. 1/2, p. 155, 1998.

[3] D. J. Sellmyer, M. Yu, and R. D. Kirby, "Nanostructured magnetic films for extremely high-density recording," Nanostructured Materials, vol. 12, p. $1021,1999$.

[4] D. Weller et al., "High $K_{u}$ materials approach to $100 \mathrm{Gbits} / \mathrm{in}^{2}$," IEEE Trans. Magn., vol. 36, no. 1, pp. 10-15, Jan. 2000.

[5] D. J. Sellmyer, C. P. Luo, M. L. Yan, and Y. Liu, "High-anisotropy nanocomposite films for magnetic recording," IEEE Trans. Magn., vol. 37, no. 4, pp. 1286-1291, Jul. 2001

[6] A. Moser and D. Weller, "Thermal effects in high-density recording media," in The Physics of Ultra-High-Density Magnetic Recording, M. L. Plumer, J. van Ek, and D. Weller, Eds. Berlin, Germany: Springer, 2001, p. 144.

[7] R. Victora, "Perpendicular recording media," in The Physics of UltraHigh-Density Magnetic Recording, M. L. Plumer, J. van Ek, and D. Weller, Eds. Berlin, Germany: Springer, 2001, p. 230.

[8] "Proceedings of the Ninth Joint MMM/Intermag Conference," J. Appl. Phys., pt. 2, vol. 95, 2004.

[9] R. H. Victora, "Areal density limits for perpendicular magnetic recording," IEEE Trans. Magn., vol. 38, no. 5, pp. 1886-1891, Sep. 2002.

[10] K.-Z. Gao and H. N. Bertram, "Magnetic recording configuration for densities beyond $1 \mathrm{~Tb} / \mathrm{in}^{2}$ and data rates beyond $1 \mathrm{~Gb} / \mathrm{s}$," IEEE Trans. Magn., vol. 38, no. 6, pp. 3675-3683, Nov. 2002.

[11] N. Honda, K. Ouchi, and S. I. Iwasaki, "Design consideration of ultrahigh-density perpendicular magnetic recording media," IEEE Trans. Magn., vol. 38, no. 4, pp. 1615-1621, Jul. 2002.

[12] J. J. Miles, D. McKirdy, R. W. Chantrell, and R. Wood, "Parametric optimization for terabit perpendicular recording," IEEE Trans. Magn., vol. 39, no. 4, pp. 1876-1890, Jul. 2003.

[13] D. J. Sellmyer, C. P. Luo, Y. Qiang, and J. P. Liu, "Magnetism of nanophase composites," in Handbook of Thin Film Materials, H. S. Nalwa, Ed. New York: Academic, 2002, vol. 5, p. 337.

[14] M. L. Yan, N. Powers, and D. J. Sellmyer, "Highly oriented nonepitaxial grown L1 $1_{0}$ FePt film,” J. Appl. Phys., vol. 93, no. 10, p. 8292, 2003.

[15] H. Zeng, M. L. Yan, N. Powers, and D. J. Sellmyer, "Orientation-controlled nonepitaxial L1 $1_{0} \mathrm{CoPt}$ and FePt films," Appl. Phys. Lett., vol. 80, no. 13, p. 2350, 2002.

[16] M. L. Yan, H. Zeng, N. Powers, and D. J. Sellmyer, "L1 $1_{0}$, (001)-oriented FePt: $\mathrm{B}_{2} \mathrm{O}_{3}$ composite films for perpendicular recording," J. Appl. Phys., vol. 91, no. 10, p. 8471, 2002.

[17] Y. Shao, M. L. Yan, and D. J. Sellmyer, "Effects of rapid thermal annealing on nanostructure, texture and magnetic properties of granular FePt:Ag films for perpendicular recording," J. Appl. Phys., vol. 93, no. 10, p. 8152, 2003.

[18] M. L. Yan, X. Z. Li, L. Gao, S. H. Liu, D. J. Sellmyer, R. J. M. van de Veerdonk, and K. W. Wierman, "Fabrication of nonepitaxially-grown, double-layered $\mathrm{FePt}: \mathrm{C} / \mathrm{FeCoNi}$ thin films for perpendicular recording,' Appl. Phys. Lett., vol. 83, no. 16, p. 3332, 2003.

[19] Y. Xu, Z. G. Sun, Y. Qiang, and D. J. Sellmyer, "Magnetic properties of $\mathrm{L}_{0}$ FePt and FePt:Ag nanocluster films," J. Appl. Phys., vol. 93, p. 8289, 2003.

[20] - "Preparation and magnetic properties of CoPt and CoPt:Ag nanocluster films," J. Magn. Magn. Mater, vol. 266, p. 164, 2003.

[21] Y. Xu and D. J. Sellmyer, unpublished work.

[22] Y. Xu, M. L. Yan, and D. J. Sellmyer, "Nanostructure and magnetic properties of FePt:C cluster films," IEEE Trans. Magn., vol. 40, no. 4, pp. 2525-2527, Jul. 2004.

[23] M. P. Sharrock, "Time dependence of switching fields in magnetic recording media,” J. Appl. Phys., vol. 76, p. 6413, 1994.

[24] Y. Xu, Z. S. Shan, J. P. Wang, and C. T. Chong, "Magnetic and reversal properties of HCP-CoCrPt:C granular films with CrTi underlayer," $J$. Magn. Magn. Mater, vol. 232, p. 103, 2001. 